\title{
Limiar de Variabilidade da Freqüência Cardíaca em Adolecentes Obesos e Não-Obesos
}

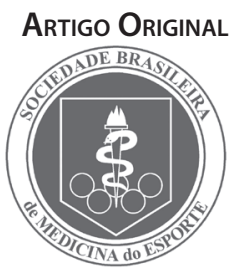

\author{
Heart Rate Variability Threshold in Obese and Non-Obese Adolescents
}

\author{
Antônio Fernando Brunetto ${ }^{1}$ \\ Bruno Tesini Roseguini ${ }^{1}$ \\ Bruno Moreira Silva ${ }^{1}$ \\ Daniel Müller Hirai $^{1}$ \\ Enio Vaz Ronque 2 \\ Dartagnan Pinto Guedes² \\ 1. Departamento de Fisioterapia \\ - Universidade Estadual de Londrina \\ 2. Departamento de Educação Física \\ - Universidade Estadual de Londrina
}

\section{Endereço para correspondência:}

Rua Vasco da Gama 128 - Aeroporto CEP 86036-010 - Londrina - Paraná

Email: brunetto@uel.br

Submetido em 15/12/2005

Versão final recebida em 19/12/2006

Aceito em 22/12/2006

\begin{abstract}
RESUMO
A obesidade na adolescência está associada à disfunção simpato-vagal cardíaca em repouso, embora existam poucas informações sobre a resposta autonômica durante o exercício nestes adolescentes. Objetivo: Comparar a modulação autonômica durante teste de esforço físico dinâmico incremental em amostras de adolescentes obesos e não-obesos, e analisar a relação entre o limiar de variabilidade da freqüência cardíaca (LiVFC) e o limar ventilatório (LV). Métodos: Dez adolescentes obesos e 19 adolescentes não-obesos do sexo masculino com idades entre 13 e 18 anos foram submetidos à teste de esforço físico progressivo máximo em esteira rolante para estudo da variabilidade da freqüência cardíaca (VFC) e para identificação do LV. A VFC foi estudada mediante análise do desvio-padrão da variabilidade instantânea batimento-abatimento (SD1) da Plotagem de Poincaré. O LiVFC foi identificado na intensidade de esforço físico em que o SD1 atingiu valor menor que $3 \mathrm{~ms}$. Resultados: O índice SD1 diminuiu progressivamente em ambos os grupos até aproximadamente $50-60 \%$ do $\mathrm{VO}_{2 \text { pico, }}$ sendo que os adolescentes obesos apresentaram valores significativamente menores $(p<0,001)$ somente na intensidade relativa a $20 \%$ do $V_{2} O_{2 \text { icor }}$ Os adolescentes obesos apresentaram valores relativos $\left(\mathrm{mL}^{\mathrm{k}} \mathrm{kg}^{-1} \cdot \mathrm{min}^{-1}\right)$ de $\mathrm{VO}_{2 \text { pico }}$ e LV significativamente menores $(p<0,05)$ que os adolescentes não-obesos. Em ambos os grupos, não foram observadas diferenças significativas entre o ponto de ocorrência do LV e do LiVFC, expressos em valores relativos e absolutos de $\mathrm{VO}_{2}$. Contudo, não foram encontradas correlações significativas entre o ponto de ocorrência do LV e do LiVFC, tanto em valores relativos quanto em valores absolutos de $\mathrm{VO}_{2}$. Conclusões: A obesidade na adolescência parece não estar associada à alteração da modulação autonômica durante o exercício físico. Torna-se possível determinar o LiVFC mediante análise da Plotagem de Poincaré em adolescentes obesos e não-obesos. Entretanto, a ausência de associação estatística entre o LiVFC e o LV demonstra que parece não haver relação causal entre estes eventos.
\end{abstract}

Palavras-chave: sistema nervoso autônomo, variabilidade da freqüência cardíaca, obesidade, exercício físico, limiar ventilatório.

\section{ABSTRACT}

Obesity in adolescence is associated with a cardiac sympathetic-parasympathetic dysfunction at rest. However, there is little information about the autonomic response during exercise in these adolescents. Objective: To compare the cardiac autonomic modulation during a maximal exercise test in obese and nonobese adolescents and analyze the association between the Heart Rate Variability threshold (HRVT) and the Ventilatory threshold (VT). Methods: Ten obese and 19 non-obese male adolescents with age between 13-18 years were submitted to a maximal progressive treadmill physical exercise test for Heart Rate Variability (HRV) study and VT determination. HRV was assessed through the study of Poincare Plot, by the analysis of the standard deviation of instantaneous beat-to-beat variability (SD1). HRVT was defined as the effort intensity at which the SD1 reached less than 3 ms. Results: The SD1 index reduced progressively in both groups until approximately $50-60 \%$ of $\mathrm{VO}_{2 \text { peak }}$ while the obese adolescents showed lower significant values $(p<0.001)$ only in the relative intensity of $20 \%$ of $\mathrm{VO}_{2 \text { peak }}$. The obese adolescents showed relative values $\left(\mathrm{mL}^{\mathrm{kg}}{ }^{-1} \cdot \mathrm{min}^{-1}\right)$ of $\mathrm{VO}_{2 \text { peak }}$ and $\mathrm{VT}$ significantly lower $(\mathrm{p}<0.05)$ than non-obese ones. There were no significant differences between VT and HRVT, expressed in absolute and relative $\mathrm{VO}_{2}$ values for neither groups. However, no significant correlations between VT and HRVT were found, neither in absolute nor relative values. Conclusions: Obesity in adolescence does not seem to be associated with the autonomic modulation during physical exercise. It is possible to determine the HRVT by Poincaré Plot in obese and non-obese adolescents. Nonetheless, the absence of statistical association between HRVT and VT suggests a lack of causal relationship between these events.

Keywords: autonomic nervous system, heart rate variability, obesity, physical exercise, ventilatory threshold. 


\section{INTRODUÇÃO}

A análise da variabilidade da freqüência cardíaca (VFC) tem se mostrado útil para avaliar o sistema nervoso autônomo durante testes de esforço físico dinâmico incremental (TEF) ${ }^{(1-5)}$. Durante a prática do exercício físico o aumento inicial da freqüência cardíaca é determinado predominantemente pela retirada da atividade parassimpática, seguida por uma marcante ativação simpática( ${ }^{(6)}$. Segundo observações de Tulppo et al $\left.\right|^{(7,8)}$ e de Yamamoto et $a^{\left({ }^{(4)}\right.}$, a atividade parassimpática decresce progressivamente até sua retirada quase completa em aproximadamente 50 a $60 \%$ do $\mathrm{VO}_{2 \text { pico, }}$ estando este evento autonômico aparentemente relacionado à ocorrência do primeiro limar ventilatório (LV). Esta intensidade do esforço físico, correspondente ao término da expressiva retirada da atividade vagal, vem sendo denominada na literatura científica de limiar de variabilidade da freqüência cardíaca $\left(\right.$ LiVFC $^{(2)}$.

Em adolescentes obesos, o estudo da VFC em repouso tem mostrado que estes indivíduos apresentam uma disfunção simpato-vagal cardíaca, caracterizada por redução da atividade parassimpática e possivelmente aumento da atividade simpática ${ }^{(9,10)}$, associados, aparentemente, à disfunção dos mecanismos de controle baroreflexo da freqüência cardíaca ${ }^{(11)}$. No entanto, pelo melhor do nosso conhecimento, nenhum estudo até o momento investigou o impacto da obesidade na adolescência sobre a resposta autonômica durante a realização de esforços físicos, assim como, sobre a relação entre a resposta autonômica e as respostas metabólicas. Dessa forma, os objetivos do presente estudo foram comparar a modulação autonômica durante teste de esforço físico dinâmico incremental em amostras de adolescentes obesos e não-obesos, e analisar a relação entre o LiVFC e o LV.

\section{MÉTODOS}

Foram analisados 10 rapazes obesos e 19 rapazes não-obesos, com idades entre 13 e 18 anos, recrutados em escolas públicas locais que não apresentavam experiência anterior em treinamento esportivo sistematizado. Todos os sujeitos completaram um questionário a respeito da história médica pregressa, consumo de bebidas alcoólicas, uso de tabaco e nível de prática habitual de atividade física. Foram excluídos da amostra os adolescentes que apresentavam história de doenças cardiovasculares e pulmonares, diabetes, síncope ou que relataram estar utilizando qualquer tipo de medicamento. Os adolescentes e seus pais e/ou responsáveis foram previamente informados quanto aos procedimentos experimentais empregados e apresentaram, por escrito, consentimento para participação. Os protocolos de intervenção foram aprovados pelo Comitê de Ética em Pesquisa da Universidade Estadual de Londrina e acompanham normas da Resolução 196/96 do Conselho Nacional de Saúde sobre pesquisa envolvendo seres humanos.

Após mensuração do peso corporal e da estatura foi calculado o índice de massa corporal (IMC) dos adolescentes, mediante a razão entre as medidas do peso corporal expresso em quilogramas e da estatura expressa em metros ao quadrado $\left(\mathrm{kg} / \mathrm{m}^{2}\right)$. Para identificação da obesidade foram adotados os pontos de corte para sexo e idade sugeridos no estudo promovido pela Força Tarefa Internacional para Obesidade ${ }^{(12)}$. As características físicas dos adolescentes analisados são apresentadas na tabela 1.

\section{Teste de esforço físico dinâmico incremental (TEF)}

Nos dias em que foram coletadas as informações para o estudo, os adolescentes foram instruídos a não consumir bebidas a base de cafeína e nenhum tipo de bebida alcoólica. Os adolescentes analisados também foram orientados no sentido de não realizarem atividades físicas extenuantes a partir do dia anterior à coleta de dados. Todos os
Tabela 1. Médias e desvios-padrão das características antropométricas dos adolescentes analisados.

\begin{tabular}{l|c|c|c}
\hline & $\begin{array}{c}\text { Não-Obesos } \\
(\mathbf{n}=\mathbf{1 9})\end{array}$ & $\begin{array}{c}\text { Obesos } \\
(\mathbf{n = 1 0 )}\end{array}$ & $\mathbf{p}$ \\
\hline Idade (anos) & $15,6 \pm 1,0$ & $15,1 \pm 1,0$ & $\mathrm{~ns}$ \\
\hline Peso Corporal $(\mathrm{kg})$ & $63,5 \pm 9,0$ & $89,6 \pm 9,5$ & 0,001 \\
\hline Estatura (cm) & $171,9 \pm 6,5$ & $174,1 \pm 7,2$ & $\mathrm{~ns}$ \\
\hline Índice de Massa Corporal $\left(\mathrm{kg} / \mathrm{m}^{2}\right)$ & $21,4 \pm 2,4$ & $29,5 \pm 2,2$ & 0,001 \\
\hline
\end{tabular}

procedimentos do estudo foram realizados entre 14:00 e 16:00 horas, após uma refeição leve, em uma sala silenciosa com temperatura ambiente entre $20^{\circ}$ e $23^{\circ} \mathrm{C}$.

Detalhes dos procedimentos metodológicos empregados foram descritos previamente ${ }^{(13)}$. Todos os sujeitos foram submetidos a um teste de esforço físico máximo (TEF), sintoma-limitado, em esteira ergométrica (Inbrasport/Millennium, Porto Alegre, Brasil). Previamente ao início do TEF foi oportunizado aos adolescentes um período de adaptação à prática de caminhada/corrida na esteira. Em seguida, após um período de descanso, foi iniciado o TEF utilizando-se o protocolo de Bruce com incremento progressivo de carga de trabalho a cada três minutos. Incentivo verbal foi empregado na tentativa de obter um esforço físico próximo do máximo. O teste foi interrompido mediante exaustão voluntária. Em repouso e durante o TEF foram continuamente registrados o volume minuto $(\mathrm{VE})$, o consumo de oxigênio $\left(\mathrm{VO}_{2}\right)$ e a produção de dióxido de carbono $\left(\mathrm{VCO}_{2}\right)$ pela análise de trocas gasosas pulmonares (analisador metabólico VO2000 - Medical Graphics, St. Paul, MN, EUA). A partir dessas informações, foram estabelecidas a razão de troca respiratória $\left(\mathrm{R}=\mathrm{VCO}_{2} \mathrm{NO}_{2}\right)$, o equivalente ventilatório de oxigênio $\left(\mathrm{VENO}_{2}\right)$ e o equivalente ventilatório de dióxido de carbono $\left(\mathrm{VE} / \mathrm{NCO}_{2}\right)$. As variáveis ventilatórias foram coletadas a cada minuto. O equipamento para análise de trocas gasosas foi calibrado previamente ao início de cada TEF, conforme padronização do fabricante.

$\mathrm{O} \mathrm{VO}_{2 \text { pico }}$ foi estabelecido como o consumo de oxigênio mais elevado alcançado durante o TEF. O LV foi identificado na intensidade de esforço físico em que o VENO $\mathrm{Na}_{2}$ atingiu seu valor mínimo antes de apresentar aumentos progressivos, sem que ocorressem aumentos concomitantes do $\mathrm{VE} / \mathrm{VCO}_{2}{ }^{(14)}$. Quando a resposta de $\mathrm{VE} / \mathrm{NO}_{2}$ não fornecia de forma inequívoca o LV, foi utilizado o método V-Slope para confirmação ${ }^{(15)}$. O LV foi determinado independentemente por três diferentes avaliadores, sendo que a mediana dos valores fornecidos pelos avaliadores foi assumida como o LV.

\section{Mensuração dos intervalos R-R e análise da Variabilidade da Freqüência Cardíaca (VFC)}

Os intervalos R-R foram continuamente registrados batimento-abatimento por um freqüencímetro cardíaco (Polar S810, Polar Electro, Kempele, Finlândia) em repouso e durante o TEF ${ }^{(16)}$. Os dados foram coletados com freqüência de amostragem de $1000 \mathrm{~Hz}$. Os registros dos intervalos R-R foram editados manualmente através de inspeção visual na tentativa de evitar que artefatos contaminassem a análise. Na seqüência, os registros foram automaticamente filtrados pelo software Polar Precision Performance (versão 3.02.007). Qualquer intervalo R-R com diferença superior a 20\% do intervalo anterior foi automaticamente filtrado ${ }^{(4)}$.

A análise da VFC foi realizada mediante análise quantitativa da Plotagem de Poincaré, que consiste em um diagrama onde cada intervalo R-R é plotado em função do intervalo anterior. O desvio-padrão da variabilidade instantânea batimento-a-batimento (SD1), foi utilizado 
como marcador da atividade parassimpática no repouso e durante o esforço físico (7). Os intervalos R-R foram agrupados em seqüências de um minuto para análise da VFC, sendo que o primeiro minuto de esforço físico foi excluído da análise devido ao brusco aumento da freqüência cardíaca e redução transitória da VFC, atribuídos à súbita retirada vagal(6). Acompanhando proposição de Lima e Kiss ${ }^{(2)}$, o LiVFC foi definido mediante a intensidade de esforço físico em que o índice SD1 atingiu valor menor que 3 ms.

\section{Análise Estatística}

Os valores a cada minuto do índice SD1 e dos intervalos R-R foram interpolados a $0,08 \mathrm{~Hz}$ (software Microcal Origin 6.0) na tentativa de relatar as informações associadas à VFC de acordo com o $\mathrm{VO}_{2}$ relativo das intensidades de esforço correspondentes a 20, 30, 40, 50, 60, 70, 80,90 e $100 \%$ do $\mathrm{VO}_{2 \text { pico. }}$.

Todos os procedimentos estatísticos foram realizados utilizando-se do pacote estatístico Statistical Package for the Social Science, versão 13.0 para Windows (SPSS Inc, Chicago, IL). A distribuição normal dos dados foi verificada pelo teste de Shapiro Wilk. Para comparação das características antropométricas e do ponto de ocorrência do LV e do LiVFC entre os rapazes obesos e não-obesos foram utilizados os procedimentos da análise de variância One-Way para amostras independentes. O coeficiente de correlação momento-produto de Pearson foi empregado para análise das relações estatísticas entre os limiares. A comparação dos índices SD1 e dos intervalos R-R durante o esforço físico entre os grupos, em proporção ao $\mathrm{VO}_{2 p i c o}$, foi realizada mediante os procedimentos da análise de variância Two-Way para medidas repetidas. Quando as análises de variâncias identificaram diferenças significativas, empregou-se o teste de comparações múltiplas post hoc de Scheffé.

\section{RESULTADOS}

A figura 1 mostra o comportamento observado nos intervalos R-R e no índice SD1 em proporção ao $\mathrm{VO}_{2 \text { pico. }}$. Como observado, o índice SD1 diminuiu progressivamente em ambos os grupos, até aproximadamente $50-60 \%$ do $\mathrm{VO}_{2 \text { picor }}$ atingindo um platô em valores próximos a $3 \mathrm{~ms}$. Ocorreram diferenças significativas entre os grupos somente na intensidade relativa a $20 \%$ do $\mathrm{VO}_{2 \text { pico, }}$ sendo que os adolescentes obesos apresentaram valores significativamente menores $(p<0,001)$ de SD1 e maiores $(p<0,001)$ de $R-R$ quando comparados aos adolescentes não-obesos.

As respostas cardiorrespiratórias diante do TEF estão apresentadas na tabela 2. Os valores absolutos ( $L$. $\left.\mathrm{min}^{-1}\right)$ do $\mathrm{VO}_{2 \text { pico }}$ e do $\mathrm{LV}$ foram significativamente maiores $(p<0,01)$ nos adolescentes obesos quando comparados aos não-obesos. Contudo, quando expresso em valores relativos à massa corporal $\left(\mathrm{mL} \mathrm{kg}^{-1} \cdot \mathrm{min}^{-1}\right)$, os valores médios do $\mathrm{VO}_{2 \text { pico }}$ e do LV foram significativamente $(p<0,05)$ menores nos adolescentes obesos. Da mesma forma, o valor médio do $\mathrm{VO}_{2}$ no ponto de ocorrência do LiVFC foi significativamente maior $(p<0,05)$ nos adolescentes obesos quando expresso em valores absolutos. Entretanto, em valores relativos, não ocorreram diferenças significativas entre os grupos.

Nas análises intragrupos não foram observadas diferenças significativas entre o ponto de ocorrência do LV e o do LiVFC, expressos em valores relativos ou absolutos do $\mathrm{VO}_{2}$. Nos adolescentes não-obesos o LV e o LiVFC foram identificados, respectivamente, em aproximadamente $57 \%$ e $54 \%$ do $\mathrm{VO}_{2 \text { pico. }}$. No grupo dos adolescentes obesos o LV foi identificado em torno de $59 \%$ do $\mathrm{VO}_{2 \text { picor }}$ enquanto o LiVFC foi identificado em torno de $54 \%$ do $\mathrm{VO}_{2 \text { pico. }}$. Contudo, não foram encontradas correlações significativas entre o ponto de ocorrência do LV e do LiVFC, em ambos os grupos, tanto em valores relativos quanto em
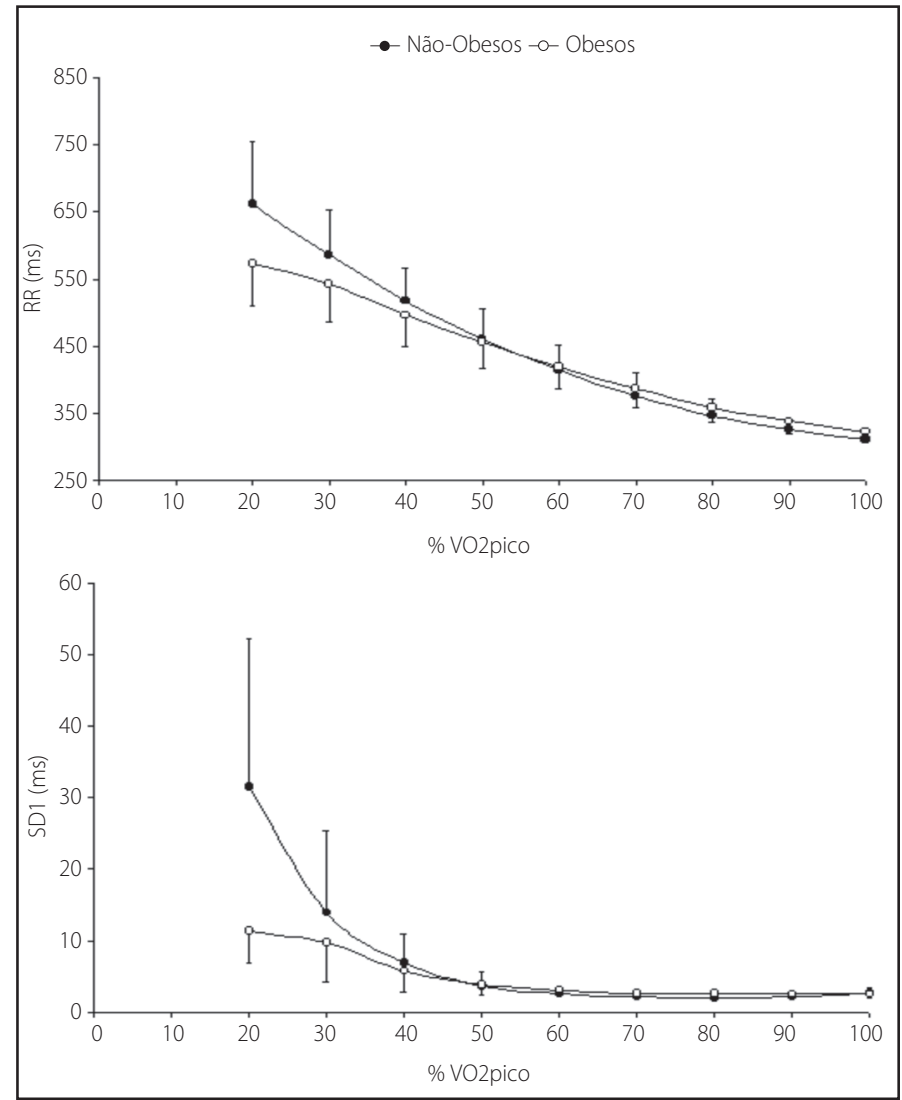

Figura 1. Comportamento dos intervalos R-R e do índice SD1 (desvio-padrão da variabilidade instantânea batimento-a-batimento) durante a realização de esforço físico relatado em proporção do $\mathrm{VO}_{2 \text { pico. }}{ }^{*} p<0.05$ obesos vs não-obesos.

Tabela 2. Respostas cardiorrespiratórias de adolescentes obesos e não-obesos diante de teste de esforço físico máximo em esteira rolante.

\begin{tabular}{|c|c|c|c|}
\hline & $\begin{array}{c}\text { Não-Obesos } \\
(n=19)\end{array}$ & $\begin{array}{l}\text { Obesos } \\
(n=10)\end{array}$ & p \\
\hline $\mathrm{VO}_{2 \text { pico }}\left(\mathrm{mL} . \mathrm{kg}^{-1} \cdot \mathrm{min}^{-1}\right)$ & $45,7 \pm 7,6$ & $39,0 \pm 4,7$ & 0,05 \\
\hline $\mathrm{VO}_{2 \text { pico }}\left(\mathrm{L}_{\mathrm{m}} \mathrm{min}^{-1}\right)$ & $2,94 \pm 0,43$ & $3,46 \pm 0,38$ & 0,01 \\
\hline FCmáx (bpm) & $196 \pm 7$ & $191 \pm 8$ & ns \\
\hline $\mathrm{LV}\left(\mathrm{VO}_{2}-\mathrm{mL} \cdot \mathrm{kg}^{-1} \cdot \mathrm{min}^{-1}\right)$ & $26,0 \pm 3,7$ & $23,0 \pm 2,2$ & 0,05 \\
\hline $\operatorname{LV}\left(\mathrm{VO}_{2}-\operatorname{L} \cdot \mathrm{min}^{-1}\right)$ & $1,64 \pm 0,31$ & $2,06 \pm 0,31$ & 0,01 \\
\hline LiVFC $\left(\mathrm{VO}_{2}-\mathrm{mL} \cdot \mathrm{kg}^{-1} \cdot \mathrm{min}^{-1}\right)$ & $24,1 \pm 4,4$ & $21,1 \pm 6,1$ & ns \\
\hline LiVFC $\left(\mathrm{VO}_{2}-\right.$ L. $\left.\mathrm{min}^{-1}\right)$ & $1,5 \pm 0,3$ & $1,9 \pm 0,7$ & 0,05 \\
\hline FC LV (bpm) & $141 \pm 13$ & $150 \pm 13$ & ns \\
\hline FC LiVFC (bpm) & $138 \pm 11$ & $147 \pm 133$ & 0,05 \\
\hline
\end{tabular}

valores absolutos do $\mathrm{VO}_{2}$. A freqüência cardíaca no ponto de ocorrência do LiVFC foi significativamente maior $(p<0,05)$ no grupo obeso quando comparado ao grupo não-obeso, enquanto a FC no LV não foi diferente entre ambos os grupos.

Mediante uma análise individual, observou-se que, em 16 adolescentes o LiVFC precedeu o LV; em 8 adolescentes o mesmo aconteceu após o LV, e em 5 adolescentes tanto o LiVFC como o LV foram identificados em valores de $\mathrm{VO}_{2}$ relativo semelhantes. A figura 2 ilustra a relação entre o ponto de identificação do LV e do LiVFC em quatro adolescentes envolvidos no estudo, sendo dois obesos e dois nãoobesos. Nos indivíduos A e C são observadas coincidências quanto aos valores do $\mathrm{VO}_{2}$ relativo em que foram identificados o LV e o LiVFC. Nos indivíduos B e D, a identificação do LiVFC precedeu a do LV. 
A

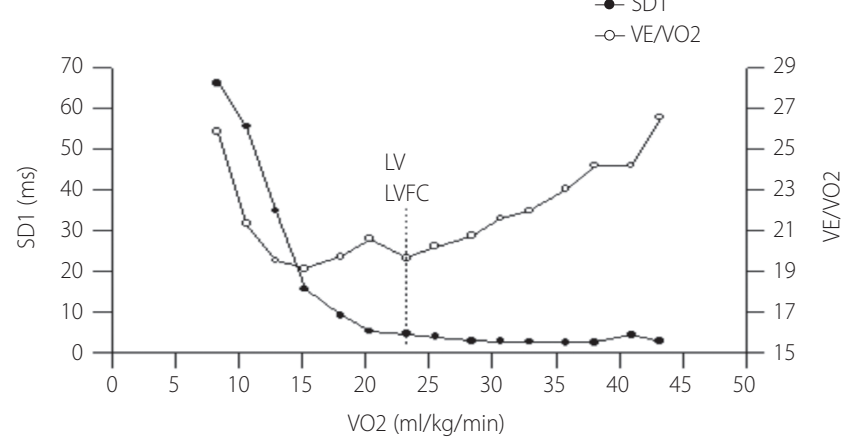

C

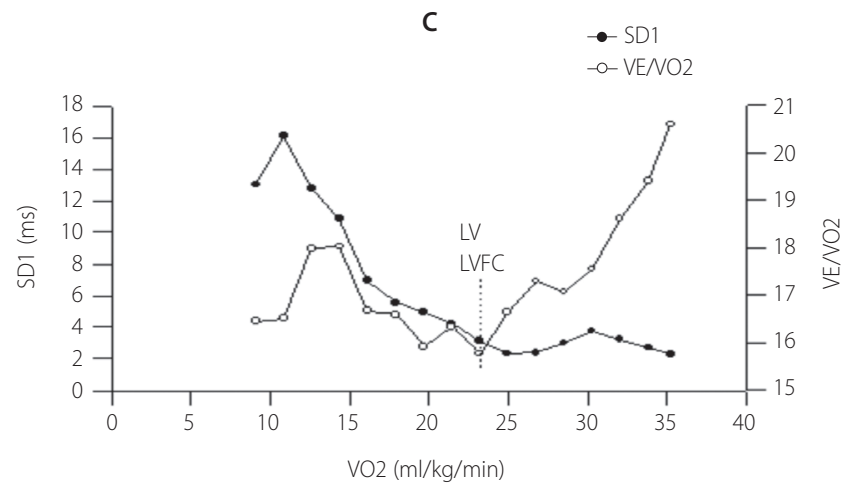

B

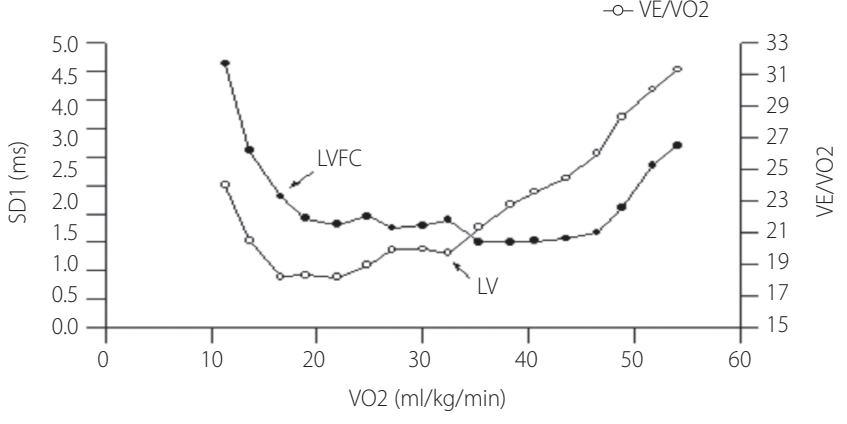

D

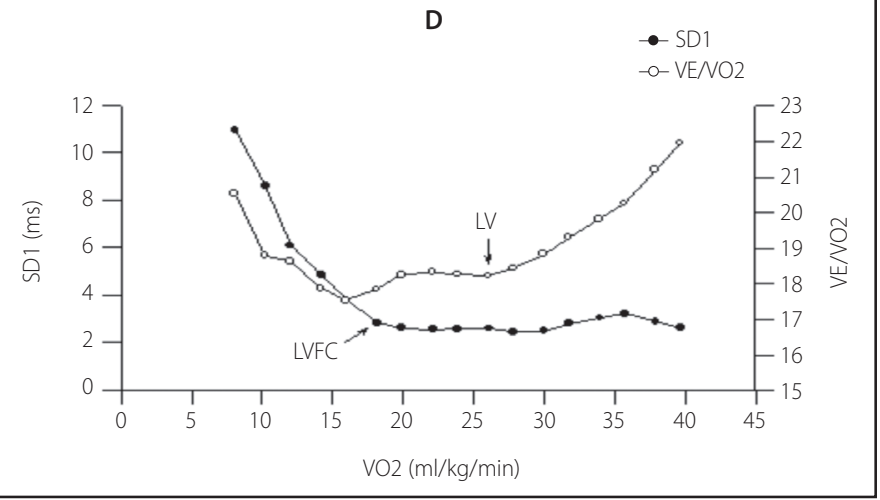

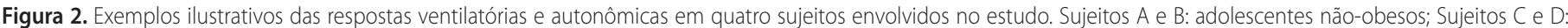
adolescentes obesos.

\section{DISCUSSÃO}

Durante o exercício físico incremental tem sido sugerido que a atividade vagal cardíaca decresce progressivamente até sua retirada quase completa em intensidades correspondentes a 50-60\% do VO 2 pico ${ }^{(4,7)}$. Da mesma forma, no presente estudo, o índice SD1, marcador da atividade vagal, diminuiu progressivamente apresentando na seqüência tendência à estabilização em aproximadamente $60 \%$ do $\mathrm{VO}_{2 \text { pico. }}$. Com exceção da intensidade relativa de $20 \%$ do $\mathrm{VO}_{2 \text { picor }}$ o índice SD1 apresentou um comportamento semelhante nos adolescentes obesos e não-obesos. A atividade parassimpática diminuída no início do exercício físico nos adolescentes obesos reflete, possivelmente, a disfunção simpato-vagal que estes adolescentes apresentam em repouso(9-11, 17).

Em um estudo prévio conduzido em nosso laboratório, com amostra semelhante à do presente estudo, foi observado que os adolescentes obesos apresentaram modulação autonômica cardíaca alterada em repouso ${ }^{(11)}$. Além disso, estes foram hiporesponsivos à manobra postural ortostática, o que sugere alteração nos mecanismos de controle baroreflexo da freqüência cardíaca. No entanto, cabe aqui destacar, que existem evidências de que a VFC em repouso e em resposta à manobra postural ortostática não está associada à condição aeróbia máxima em adolescentes sem excesso de peso corporal(18).

As respostas cardiovasculares ao exercício físico dependem de mecanismos distintos dos ajustes em repouso e frente ao estresse ortostático. Durante o exercício físico dinâmico há deslocamento do ponto operacional do baroreflexo, sendo que este passa a ter pouca participação no aumento e controle da FC. As respostas cardiovasculares passam a depender principalmente de informações aferentes provenientes de centros nervosos superiores, mecanoreceptores e quimioreceptores musculares ${ }^{(19)}$. Considerando que aparentemente estes indivíduos apresentam uma disfunção em repouso, e durante o estresse ortostático, os resultados do presente estudo adicionam informações sobre o controle cardiovascular de adolescentes obesos, mostrando ausência de alterações na modulação autonômica durante o exercício físico.

Diversas investigações vêm mostrando que parece haver uma relação entre o término da progressiva retirada vagal e a identificação do $L^{(4,5,7)}$ e do limiar de lactato(2) no exercício físico. Em nosso estudo, o LiVFC, identificado mediante análise quantitativa da Plotagem de Poincaré, ocorreu em intensidades de exercício físico e em freqüências cardíacas semelhantes a do LV em ambos os grupos. No entanto, de forma geral, os valores relacionados à ocorrência do LiVFC foram discretamente menores que os do LV. De modo semelhante, no estudo de Shibata et al(20) a freqüência cardíaca do limiar vagal foi menor que a do LV, o que levou os autores a sugerir que possivelmente a supressão da atividade vagal preceda a ocorrência do LV.

Embora estes achados suportem o fato de uma possível ligação entre a atividade autonômica e o $L V^{(5)}$, nossos resultados demonstram que não é possível estabelecer uma relação de causa-efeito entre estes eventos. Apesar do LV e do LiVFC acontecerem em intensidades semelhantes, não foram encontradas correlações entre o $\mathrm{VO}_{2}$ absoluto e relativo de ocorrência destes limiares. A observação individual mostra ainda que apesar de na maioria dos adolescentes o LiVFC preceder o LV, em vários sujeitos a resposta oposta também foi observada. Como ilustrado na figura 2, existe claramente uma dissociação entre o LiVFC e o LV, o que demonstra, possivelmente, que estes limiares, embora pareçam pertencer a uma "região" metabólica semelhante, representam eventos distintos ${ }^{(13)}$.

A discrepância entre os limiares pode ainda estar relacionada à dificuldade e, sobretudo, à subjetividade na identificação do $L V^{(21)}, \mathrm{e}$ também ao critério relativamente observacional utilizado para iden- 
tificação do LiVFC(2). Em adição, o protocolo de TEF que foi utilizado, apesar de ser muito difundido na literatura científica e na prática clínica, impõe incrementos bruscos de carga, e é iniciado com caminhada e na maioria dos casos exigiu corrida, o que dificulta a identificação de limiares por não induzir a um aumento linear da intensidade de exercício físico ${ }^{(14)}$.

No presente estudo os adolescentes obesos apresentaram menor condição física quando comparados aos adolescentes não-obesos. Em concordância com outros autores ${ }^{(22-24)}$, o VO$_{2 \text { pico }}$ e o LV, expressos em valores relativos, foram menores nos adolescentes obesos. Embora controverso(25), tem sido sugerido que um dos principais fatores responsáveis pela condição física reduzida dos adolescentes obesos seja a prática diminuída de atividade física, levando a um menor condicionamento aeróbio ${ }^{(23)}$. Associado à disfunção autonômica em repouso e ao estresse postural, os baixos níveis de condicionamento aeróbio destes jovens podem estar associados ao desenvolvimento de doenças cardiovasculares e ao aumento das taxas de morbidade e mortalidade na idade adulta ${ }^{(26,27)}$.

Em conclusão, a obesidade na adolescência parece não estar associada à alteração da modulação autonômica durante o exercício. Além disso, foi possível determinar o LiVFC mediante análise da Plotagem de Poincaré em adolescentes obesos e não obesos. Entretanto, a ausência de associação estatística entre o LiVFC e o LV demonstra não haver relação causal entre estes eventos. Mais estudos são necessários no sentido de elucidar as relações entre os limiares metabólicos e o sistema nervoso autônomo no exercício físico.

Todos os autores declararam não haver qualquer potencial conflito de interesses referente a este artigo.

\section{REFERÊNCIAS BIBLIOGRÁFICAS}

1. Alonso DO, Forjaz CLM, Rezende LO. Comportamento da freqüência cardíaca e da sua variabilidade durante as diferentes fases do exercício progressivo máximo. Arq Bras Cardiol 1998; 71: 787-92.

2. Lima JRP, Kiss MAP. Limiar de variabilidade da freqüência cardíaca. Rev Bras Ativ Fis Saúde 1999; 9: 29-38.

3. Nakamura Y, Yamamoto Y, Muraoka I. Autonomic control of heart rate during physical exercise and fractal dimension of heart rate variability. J Appl Physiol 1993; 74: 875-81.

4. Yamamoto Y, Hughson RL, Peterson J. Autonomic control of heart rate during exercise studied by heart rate variability spectral analysis. J Appl Physiol 1991; 71:1136-42.

5. Yamamoto Y, Hughson RL, Nakamura Y. Autonomic nervous system responses to exercise in relation to ventilatory threshold. Chest 1992; 101: 206S-10S.

6. Maciel BC, Gallo Jr L, Neto JAM, Filho ECL, Martins LEB. Autonomic nervous control of the heart rate during dynamic exercise in normal man. Clin Sci 1986; 71:457-60.

7. Tulppo MP, Mäkikaillio TH, Takala TES, Seppänen T, Huikuri HV. Quantitative beat-to-beat analysis of heart rate dynamics during exercise. Am J Physiol 1996; 271: H244-52.

8. Tulppo MP, Mäkikallio TH, Seppänen T, Laukkanen RT, Huikuri HV. Vagal modulation of heart rate during exercise: effects of age and physical fitness. Am J Physiol 1998; 274: H424-9.

9. Rabbia F, Silke B, Conterno A. Assessment of cardiac autonomic modulation during adolescent obesity. Obes Res 2003; 11:541-8.

10. Riva P, Martini G, Rabbia F. Obesity and autonomic function in adolescence. Clin Exp Hypertens 2001; 23: $57-67$.

11. Brunetto AF, Roseguini BT, Moreira BM, Hirai DM, Guedes DP. Respostas autonômicas cardíacas a manobra postural passiva em adolescentes obesos. Rev Assoc Med Bras 2005; 51: 256-60.

12. Cole TJ; Bellizzi MC; Flegal KM; Dietz WH. Establishing a standard definition for child overweight and obesity worldwide: international survey. BMJ 2000; 320: 1240-3.

13. Brunetto AF, Moreira BM, Roseguini BT, Hirai DM, Guedes DP. Limiar ventilatório e variabilidade da freqüência cardíaca em adolescentes. Rev Bras Med Esporte 2005; 11:22-7.

14. Ribeiro JP, Yang J, Adams RP, Kuka B, Knuttgen HG. Effect of different incremental exercise protocols on the determination of lactate and ventilatory threshold. Braz J Med Biol Res 1986; 19: 109-17.
15. Wasserman K, Hansen JE, Sue DY, Casaburi R, Whipp BJ. Principles of exercise testing and interpretation. 3 rd rev. ed. Philadelphia: Lippincott Willians \& Wilkins, 1999.

16. Kingsley M, Lewis MJ, Marson RE. Comparison of Polar 810 s and an ambulatory ECG system for RR interval measurement during progressive exercise. Int J Sports Med 2005; 26: 39-44.

17. Nagai N, Matsumoto T, Kita H, Moritani T. Autonomic nervous system activity and the state and development of obesity in Japanese school children. Obes Res 2003; 11:25-32.

18. Brunetto AF, Roseguini BT, Silva BM, Hirai DM, Guedes DP. Effects of Gender and Aerobic Fitness on Cardiac Autonomic Responses to Head-Up Tilt in Healthy Adolescents. Pediatr Cardiol. 2005; 26: 418-24.

19. Rowell LB, O'Leary DS. Reflex control of the circulation during exercise: chemoreflexes and mechanoreflexes. J Appl Physiol 1990; 69: 407-18

20. Shibata M, Moritani T, Miyawaki T, Nakao K. Exercise prescription based upon cardiac vagal activity for middle-aged obese women. Int J Obes 2002; 26: 1356-62.

21. Myers J, Ashley E. Dangerous curves. A perspective on exercise, lactate, and the anaerobic threshold. Chest 1997; 111: 787-95

22. Reybrouck T, Mertens L, Schepers D, Vinckx J, Gewilling M. Assessment of cardiorespiratory exercise function in obese children and adolescents by body mass-independent parameters. Eur J Appl Physiol 1997; 75: 478-83

23. Reybrouck T, Weymans M, Vinckx J, Stijns H, Van der Schueren, Lodeweyckx M. Cardiorespiratory function during exercise in obese children. Acta Paediatr Scand 1987; 76: 342-8.

24. Zanconato S, Baraldi E, Rigon F, Vido L, Da Dalt L, Zacchello F. Gas exchange during exercise in obese children. Eur J Pediatric 1989; 148: 614-7.

25. Cooper D, Paage J, Barstow T, Springer C. Are obese children truly unfit? Minimizing the confounding effect of body size on the exercise response. J Pediatr 1990; 116: 223-30.

26. Sandivk L, Erikssen J, Thaulow E, Erikssen G, Mundal R, Rodahl K. Physical fitness as a predictor of mortality among healthy, middle-aged norwegian men. N Engl J Med 1993; 328: 533-7.

27. Blair S, Kampert J, Kohl H. Influences of cardiorespiratory fitness and other precursors on cardiovascular disease and all-cause mortality in men and women. JAMA 1996; 276: 205-10. 